\title{
The disappearance of anomalous protons at Voyager 1 and Voyager 2 in the outer heliosphere between 1998 and 2002
}

\author{
W. R. Webber, ${ }^{1}$ F. B. McDonald, ${ }^{2}$ A. C. Cummings, ${ }^{3}$ E. C. Stone, ${ }^{3}$ B. Heikkila, ${ }^{4}$ \\ and N. Lal ${ }^{4}$
}

Received 14 February 2006; revised 4 May 2006; accepted 9 May 2006; published 26 August 2006.

[1] In 1998 and 1999 at V1 and V2 in the outer heliosphere between 55 and 75 AU, large intensities of anomalous $\mathrm{H}$ were observed with a peak in the differential energy spectrum at $\sim 25 \mathrm{MeV}$. Subsequently, in 2000 and 2001 when these spacecraft were 5-10 AU further out, these intensities were greatly reduced by 11-year modulation effects, so that by the beginning of 2002 anomalous $\mathrm{H}$ had completely disappeared beneath the $\mathrm{E}^{1.0}$ spectrum of galactic $\mathrm{H}$ at low energies. The modulation between 1998 and 2002 produced intensity changes of galactic $\mathrm{H}$ similar to those observed at the Earth in the same time period except that the changes at V1 and V2 were much smaller. The intensity change at V2 was also larger than that at V1. For anomalous H, however, the intensity changes at $\mathrm{V} 1$ and V2 were a factor of 10-20 times larger than those for galactic $\mathrm{H}$, so that by the end of 2001 the anomalous $\mathrm{H}$ intensities were less than $1 \%$ of their 1998 values. Also, during one time period in late 2001 there was a large reduction of anomalous $\mathrm{H}$ at both V1 and V2 with no corresponding change in galactic $\mathrm{H}$. These different intensity changes between galactic and anomalous $\mathrm{H}$ provide an insight into particle transport, drift, and acceleration processes in the region near the heliospheric termination shock, which is located just beyond V1. Radial gradient comparisons in 1998 and also at the times of reduced anomalous $\mathrm{H}$ intensities imply that most of the intensity reduction of anomalous $\mathrm{H}$ apparently occurred at $\sim 90$ AU or beyond. This could indicate source changes in the anomalous $\mathrm{H}$ spectra but most likely is a measure of changes in the "connection" between V1 and V2 and the source region, such as might be caused by changing drift patterns and particle entry into the heliosphere.

Citation: Webber, W. R., F. B. McDonald, A. C. Cummings, E. C. Stone, B. Heikkila, and N. Lal (2006), The disappearance of anomalous protons at Voyager 1 and Voyager 2 in the outer heliosphere between 1998 and 2002, J. Geophys. Res., 111, A08107, doi:10.1029/2006JA011669.

\section{Introduction}

[2] The anomalous components $\mathrm{H}, \mathrm{He}$, and $\mathrm{O}$, thought to have their origin as interstellar neutrals accelerated in the outer heliosphere [Fisk et al., 1974], are subject to large temporal and spatial intensity variations throughout the heliosphere during an 11-year solar modulation cycle, and also between positive and negative solar magnetic polarity cycles [e.g., Stone et al., 1997; McDonald et al., 2001; Hill et al., 2003; Webber et al., 2005]. This is especially true for anomalous $\mathrm{H}$ which we shall concentrate on in this paper. For example, in the negative polarity cycle at minimum solar modulation in 1987 , anomalous $\mathrm{H}$ was only weakly

\footnotetext{
${ }^{1}$ Department of Astronomy, New Mexico State University, Las Cruces, New Mexico, USA.

${ }^{2}$ Institute of Physical Science and Technology, University of Maryland, College Park, Maryland, USA.

${ }^{3}$ Downs Laboratory, California Institute of Technology, Pasadena, California, USA.

${ }^{4}$ NASA Goddard Space Flight Center, Greenbelt, Maryland, USA.

Copyright 2006 by the American Geophysical Union. 0148-0227/06/2006JA011669
}

detectable between $\sim 30-69 \mathrm{MeV}$ at V2 ( 23 AU) [Christian et al., 1988] with an intensity $\leq 1$ particle $/ \mathrm{cm}^{2}$ sr s $\mathrm{MeV}$ which is about the same as the intensity of galactic cosmic rays of the same energy. In the ensuing positive polarity cycle, however, anomalous $\mathrm{H}$ first reappeared in 1993 at V1 and V2 [McDonald et al., 1995; Christian et al., 1995] and continued to increase in intensity up to the minimum in modulation in 1998. By 1998 the intensity of anomalous $\mathrm{H}$ in the $30-69 \mathrm{MeV}$ range was $\sim 20$ particles/ $\mathrm{cm}^{2}$ sr's MeV at V1 (70 AU) [Stone et al., 1999] more than 20 times the maximum intensity observed in the previous cycle in 1987. Indeed during the time period from 1993 to 1998 as the Voyager spacecraft moved outward, the anomalous $\mathrm{H}$ spectrum unfolded with increasing intensities at lower energies toward a spectrum very similar to that expected at the HTS [Steenberg et al., 1999]. After 1998 the intensity of this component remained high at both V1 and V2 until early in 2000 at which time the intensity rapidly decreased by a factor of at least $10-100$, becoming undetectable below the low-energy galactic $\mathrm{H}$ component by 2002.0 at both the V1 and V2 spacecraft which were now at 84 and $67 \mathrm{AU}$, respectively. It is the purpose of this paper to discuss this disappearance of anomalous $\mathrm{H}$ in more 


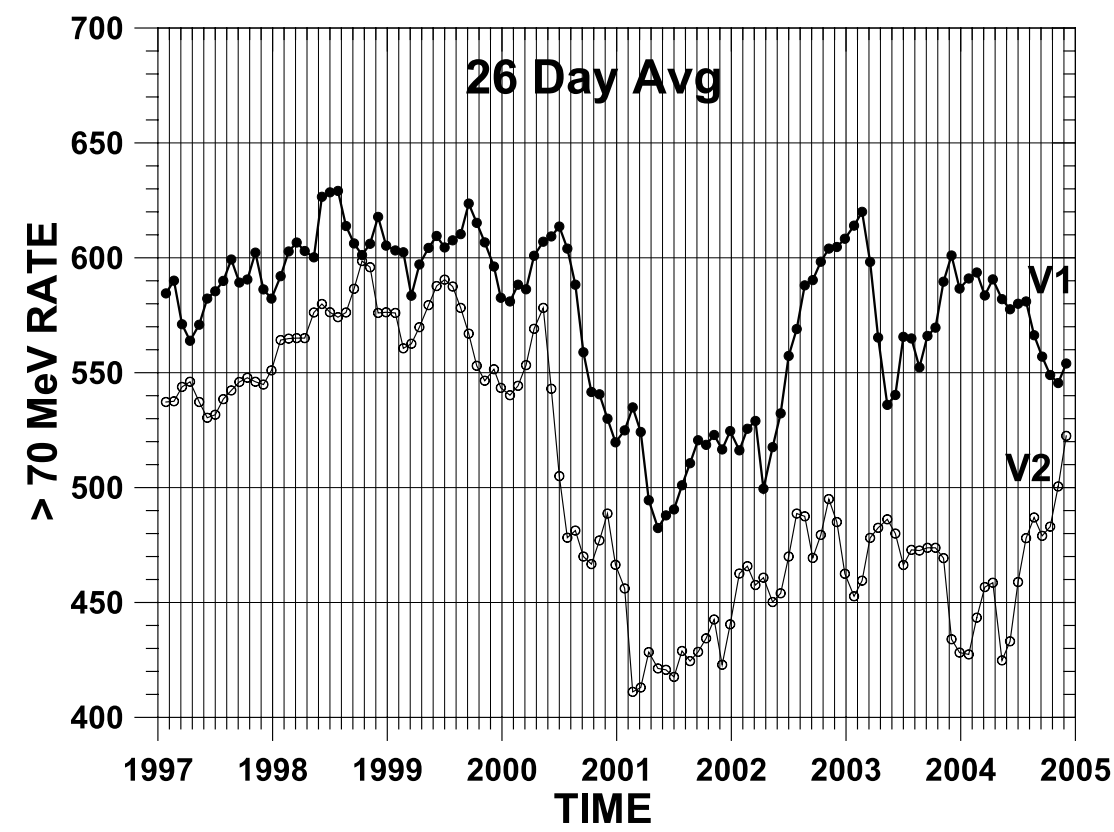

Figure 1. The 26-day average normalized rates of $>70 \mathrm{MeV}$ particles at V1 and V2 between 1997 and 2004.

detail and to compare it with the intensity decrease of galactic $\mathrm{H}$ at slightly higher energies in order to provide a basis for a theoretical understanding of these remarkable intensity changes.

\section{Data}

[3] We first look at the overall galactic cosmic ray $\mathrm{H}$ time variations at higher energies during the particular time period from $\sim 2000.0$ to 2002.0 when the anomalous $\mathrm{H}$ disappeared. We start by examining the $>70 \mathrm{MeV}$ rate from 1997 to 2004 on both V1 and V2 as displayed in Figure 1. It is seen that the intensity in this channel peaked at both spacecraft in the 1998-1999 time period, superimposed on significant time variations. At about 2000.35 at V2 and 2000.50 at V1 the onset of a large intensity decrease in the $>70 \mathrm{MeV}$ rate was observed. This decrease, which was very rapid at the beginning, continued for 0.1 to 0.2 year at both spacecraft, after which the intensity had decreased by $\sim 20 \%$ at V2 and $\sim 16 \%$ at V1. After that there is another abrupt decrease $\sim 14 \%$ at $\mathrm{V} 2$ at 2001.03 and $\sim 11.5 \%$ at V1 at 2001.19. This second decrease has earlier been related to the arrival at V2 and V1 of a large global merged interaction region from the Bastille day event at the Earth in 2000

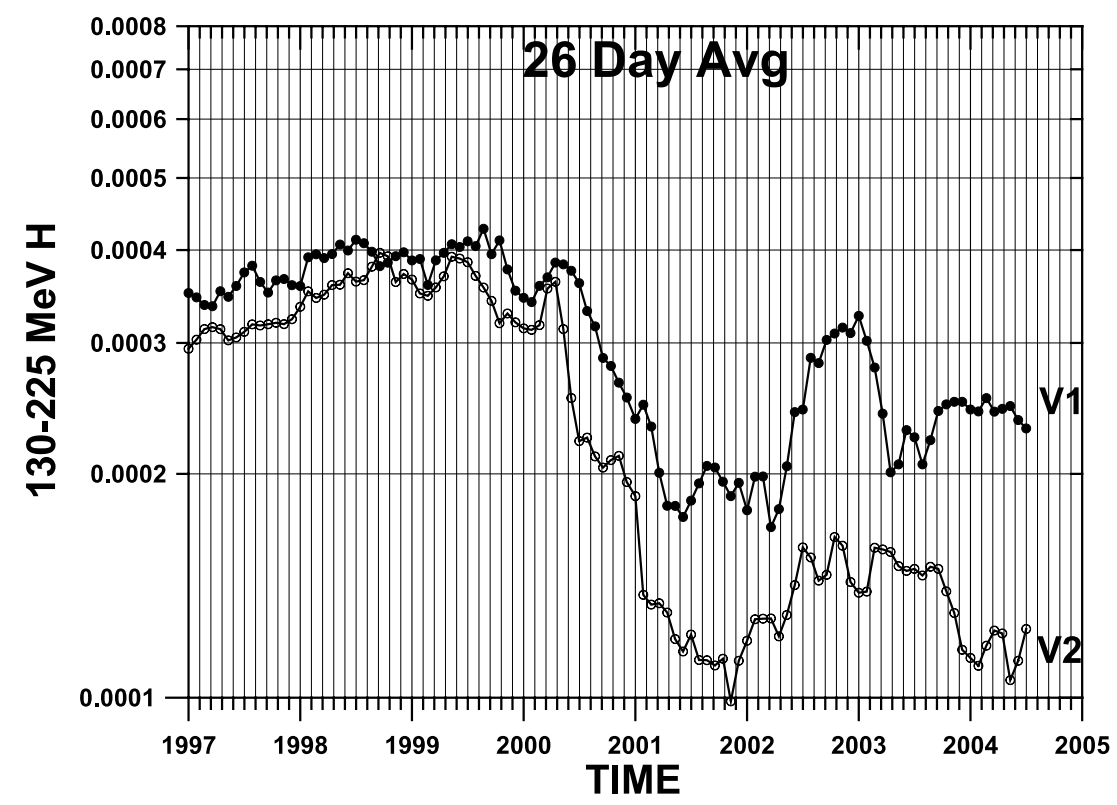

Figure 2. The 26-day average intensities of $130-240 \mathrm{MeV}$ hydrogen nuclei at V1 and V2 between 1997 and 2004. 


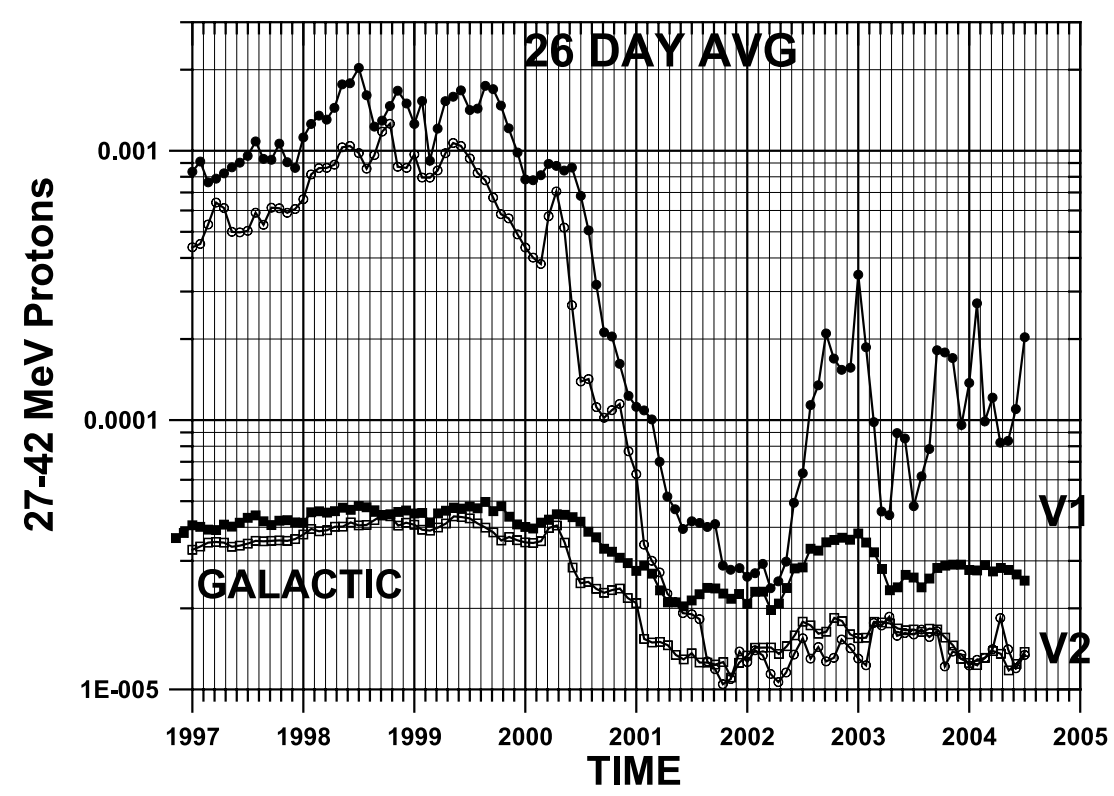

Figure 3. The 26-day average intensities of 27-42 MeV hydrogen nuclei at V1 and V2 between 1997 and 2004. Small solid squares (lower curves) show estimated galactic cosmic ray hydrogen background at V1 and V2 at this energy (see text).

[Webber et al., 2002]. After about 2002.3 the intensity of $>70 \mathrm{MeV}$ particles at both spacecraft began a slow and irregular increase from these minimum values with a large increase between 2002.6 and 2003.2 at V1 being particularly notable. In essence then the total overall decrease of $>70 \mathrm{MeV}$ particles of $\sim 32 \%$ at V2 and $\sim 23 \%$ at V1, occurred in these two steps during a time period of less than 1 year after the initial decrease began in 2000. This represents the extent and magnitude of the 11-year decrease for cycle 23 as observed for galactic cosmic rays $>70 \mathrm{MeV}$ by $\mathrm{V} 2$ and $\mathrm{V} 1$ in the outer heliosphere. This should be contrasted with the slower more complex 11-year decrease of much larger magnitude observed at the Earth between 1998 and 2001 for these same particles [e.g., McDonald et al., 2000; Webber and Lockwood, 2004a].

[4] In Figure 2 we show the 26-day average intensity variations of lower rigidity $130-240 \mathrm{MeV} \mathrm{H}$ nuclei at V1 and V2 over the time period from 1997 to 2004. These particles are also mainly galactic but contain a maximum of $10-20 \%$ contribution of anomalous cosmic ray $\mathrm{H}$ nuclei in 1998-1999. The temporal variations in this energy channel are very similar to those for $>70 \mathrm{MeV}$ particles. The amplitude of the changes for $130-240 \mathrm{MeV} \mathrm{H}$ nuclei after 1998 is larger than that for $>70 \mathrm{MeV}$ particles as would be expected because of the lower rigidity $(0.6 \mathrm{GV}$ versus $2.3 \mathrm{GV})$. The total decrease between the onset in 2000 and the minimum which occurs between 2001.5 and 2002.0 in this lower-rigidity channel is $\sim 70 \%$ at $\mathrm{V} 2$ and $\sim 52 \%$ at $\mathrm{V} 1$. This decrease is also seen to occur in two distinct steps. Again, as with the $>70 \mathrm{MeV}$ particles, the decrease during each step is largest at V2.

[5] We next turn to the anomalous $\mathrm{H}$ nuclei temporal variations during this time period and show in Figure 3 the 26-day average intensity variations of $27-42 \mathrm{MeV} \mathrm{H}$ at V1 and V2 from 1997 to 2004. For this anomalous H energy interval, the galactic particle background is of importance. This background is shown as the lower two curves in Figure 3 as determined from the $130-240 \mathrm{MeV} \mathrm{H}$ channel rate at each spacecraft assuming a $\mathrm{E}^{1.0}$ spectrum for galactic cosmic ray $\mathrm{H}$ extending to lower energies. The overall spectra of H nuclei measured at V1 and V2 in 1998 and again after all decreases have occurred at 2002.0 are shown in Figure 4. The estimated galactic particle backgrounds at low energies for the maximum intensities in 1998 and again for the minimum intensities in 2002.0 at both V1 and V2 are shown by the dashed lines in Figure 4 assuming a $\sim \mathrm{E}^{1.0}$ spectrum. In the 27-42 $\mathrm{MeV} \mathrm{H}$ channel this galactic background is seen from Figures 3 and 4 in 1998 to be only $\sim 3 \%$ of all events at V1 and $\sim 4 \%$ at V2. However, since the anomalous $\mathrm{H}\left(\equiv \mathrm{H}^{*}\right)$ intensity decreases much more rapidly at the same energy than the galactic component, this background becomes more and more significant as the intensity decreases so that by 2002.0 at both V1 and $\mathrm{V} 2$ it becomes the dominant component in the $27-42 \mathrm{MeV}$ channel.

[6] The temporal variations of the anomalous 27-42 $\mathrm{MeV} \mathrm{H}$ component itself, are very similar at both $\mathrm{V} 2$ and V1 during the time period between 2000 and 2002 to the changes during the same time period as discussed earlier for galactic cosmic rays, with the first major durable decrease of $\mathrm{H}^{*}$ at V2 starting at about 2000.3 and at V1 at about 2000.5. These temporal variations are shown in Figure 5 for the $\mathrm{H}^{*}$ component only after subtraction of galactic cosmic rays in 26-day average intervals. The initial decrease represents a factor $\sim 10$ intensity decrease at $\mathrm{V} 1$ and a factor $\sim 6.0$ intensity decrease at V2 during this time period. The intensity then remains almost constant at both spacecraft over the next 0.3 year, at which time a second sudden decrease in the intensity of $\mathrm{H}^{*}$ occurs at V2 starting at $\sim 2001.0$ and at V1 at 2001.2. Over the next 0.1-0.2 year, the intensity decreases at V2 by another factor $\sim 20$ at which 


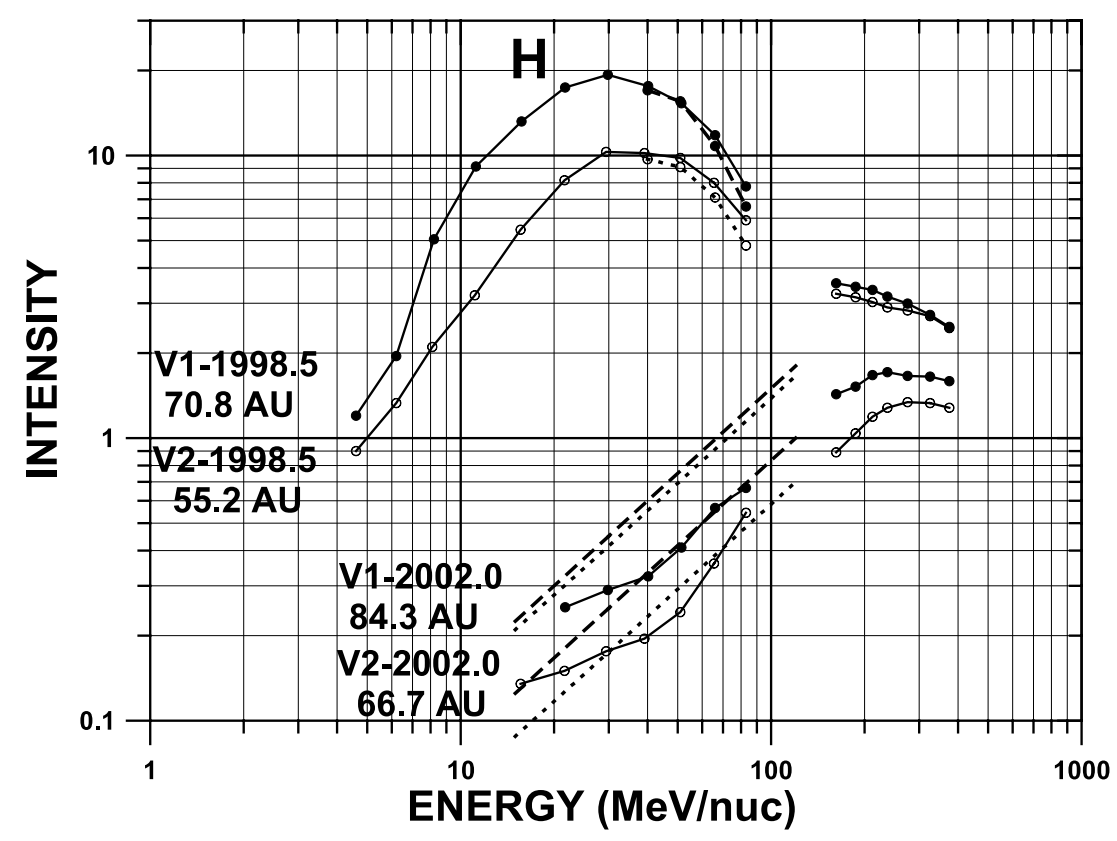

Figure 4. Measured hydrogen nuclei spectra at V1 and V2 in 1998 (maximum intensities) and at 2002.0 (minimum intensities). Pairs of dashed lines show assumed galactic hydrogen spectra $\sim \mathrm{E}^{1.0}$ at low energies at these two times.

time at 2001.4 the intensity of $\mathrm{H}^{*}$ is now only about $1 \%$ of the initial intensity in 1998. At V1 this second intensity decrease is a factor $\sim 6$ resulting in an intensity at 2001.5 which is $\sim 2 \%$ of the initial intensity in 1998 . The intensity of $27-42 \mathrm{MeV} \mathrm{H}$ then levels off at V2 between about 2001.3 and 2001.5 and at V1 between 2001.4-2001.7 before decreasing again for a third time in late 2001 so that by $2002.0, \mathrm{H}^{*}$ is essentially undetectable at both V1 and V2 in this energy channel at intensity levels less than $0.5 \%$ of the values in 1998-1999 (see also Figure 4). This third decrease is not observed in any of the galactic particle channels. The intensities in these higher-energy channels are almost constant or increase slightly during this time period. Similar intensity changes, but of a smaller magnitude, are seen at the same times for $\mathrm{He}^{*}$ and $\mathrm{O}^{*}$. These will be the subject of future papers.

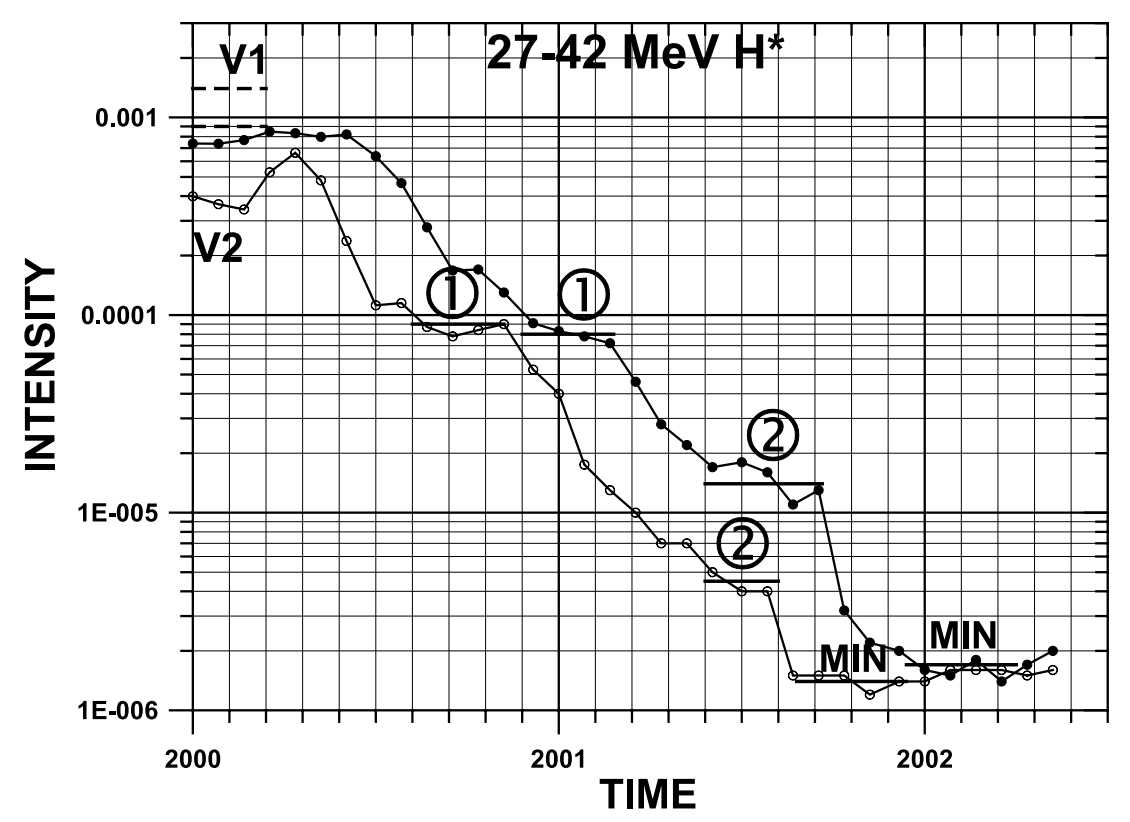

Figure 5. Decrease of $\mathrm{H}^{*}$ intensity only between 27 and $42 \mathrm{MeV}$ at V1 and V2 in 2000 and 2001. The 26-day average intensities are used. Intervals " 1 " and "2" show periods of relatively small intensity changes. Dashed lines in upper left show maximum intensities measured in 1998-1999. Intensities at MIN times are upper limits. 

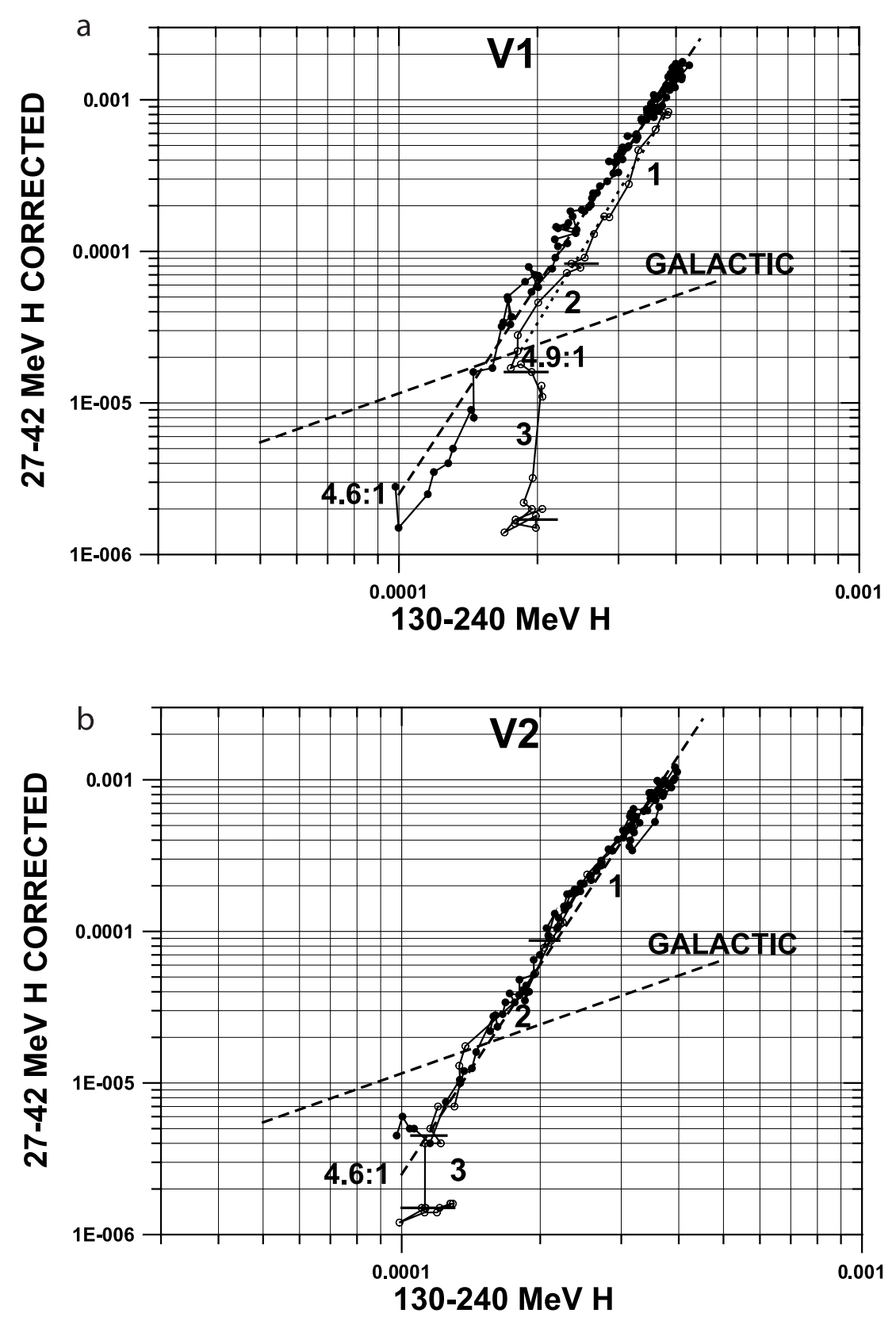

Figure 6. ( $\mathrm{a}$ and $\mathrm{b}$ ) Regression curves between 26-day average intensities of $27-42 \mathrm{MeV}$ anomalous $\mathrm{H}$ and 130-240 MeV galactic H at V1 and V2 between 1992.0 and 2002.0. Open circles are after 2000.2. Lowest-intensity $27-42 \mathrm{MeV}$ points are upper limits.

[7] In Figures 6a and 6b we show regression curves between the intensity changes seen in the $130-240 \mathrm{MeV}$ galactic $\mathrm{H}$ and the lower-energy $\mathrm{H}^{*}$ between $27-42 \mathrm{MeV}$ extending back to the intensity minimum at 1992.0. These graphs are particularly revealing. During the time period from 1992 to 1999, shown as black dots, the intensities are increasing at V1 and V2 and follow a single simple regression line. This line has a logarithmic slope $\sim 4.6: 1$ for V1. For V2 this regression line in Figure $6 \mathrm{~b}$ has a similar logarithmic slope. The first two decrease periods in 2000 and 2001, shown as open circles, are labeled 1 and 2 in Figures $6 \mathrm{a}$ and $6 \mathrm{~b}$. The dashed lines show the average regression lines between the two energies at V1 and V2.
The two decreases have similar regression lines with logarithmic slopes $\sim 4.9: 1$ for $\mathrm{V} 1$ and $\sim 4.6: 1$ for V2. Thus in less than $\sim 1$ year the intensities have decreased by a factor $\sim 100$ along a very similar regression line to the increase from 1992 to 1999.

[8] The final decrease of $\mathrm{H}^{*}$, labeled 3, which occurs in the latter half of 2001 at V1 and V2 is most unusual. The intensity at V1 decreases by an additional factor $\geq 8$ and by a factor of at least 3 at V2 and disappears below the galactic $\mathrm{H}$ background to a level 0.2 of the estimated galactic intensity at that time. This final $\mathrm{H}^{*}$ decrease is uncorrelated with the galactic $\mathrm{H}$, and in fact, during this time the higherenergy galactic particle intensities are nearly constant. 


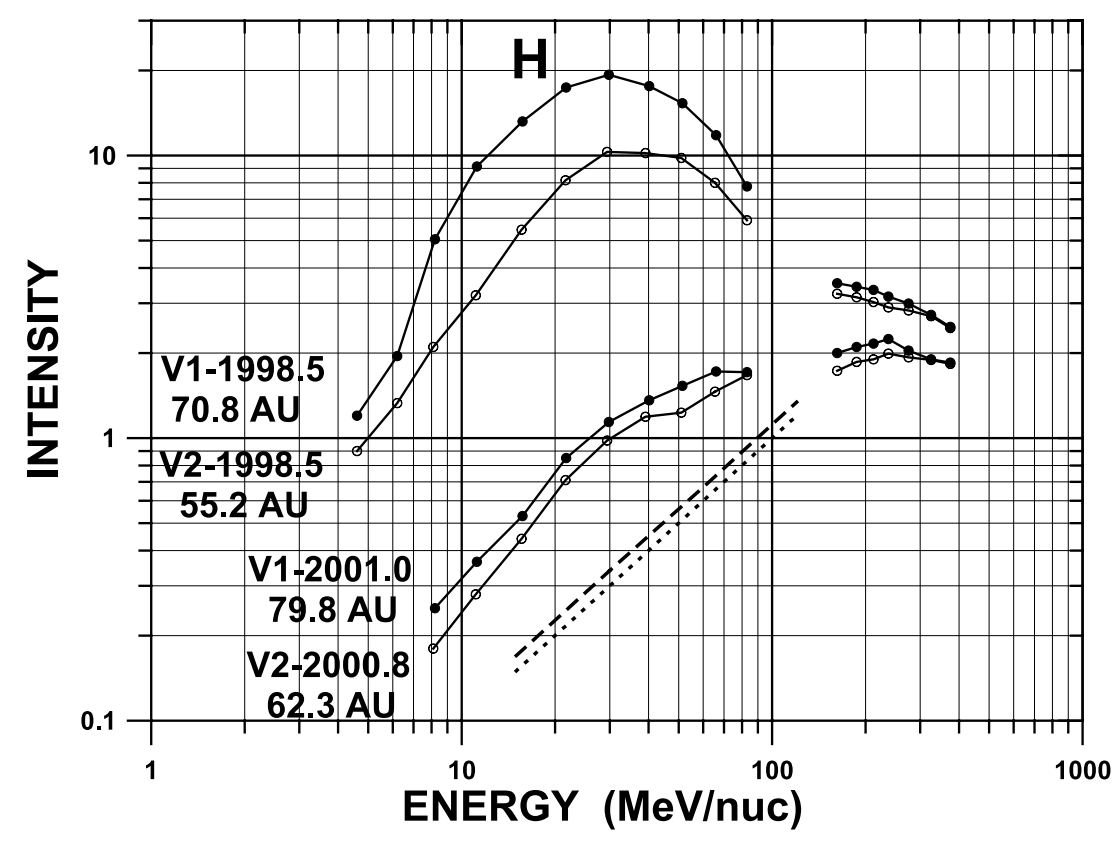

Figure 7. Measured total hydrogen spectra at V1 and V2 in 1998 and during interval "1." Pair of dashed lines are estimated low-energy galactic spectra $\sim E^{1.0}$ at V1 and V2 in interval "1."

[9] During the overall decrease that occurs from early 2000 to late 2001 there are two time periods designated as " 1 " and " 2 " in Figure 5 when the intensity changes are small at both V1 and V2. The fact that the intensity changes are small over these two periods of time between $\sim 2000.60$ and 2000.90 and again between 2001.30 and 2001.60 at V2 and roughly one 52-day period later at V1, allow us to derive energy spectra for these specific two time periods at the two spacecraft. These energy spectra are shown for time interval " 1 " in Figure 7 along with the initial spectra for the 1998 time period and in Figure 8 for time interval " 2 " along with the initial spectra in 1998.

\section{Modulation of Galactic and Anomalous $H$ Nuclei Between 1998 and 2002}

[10] It is clear that along with a much smaller modulation of galactic cosmic rays, there was a very large reduction of

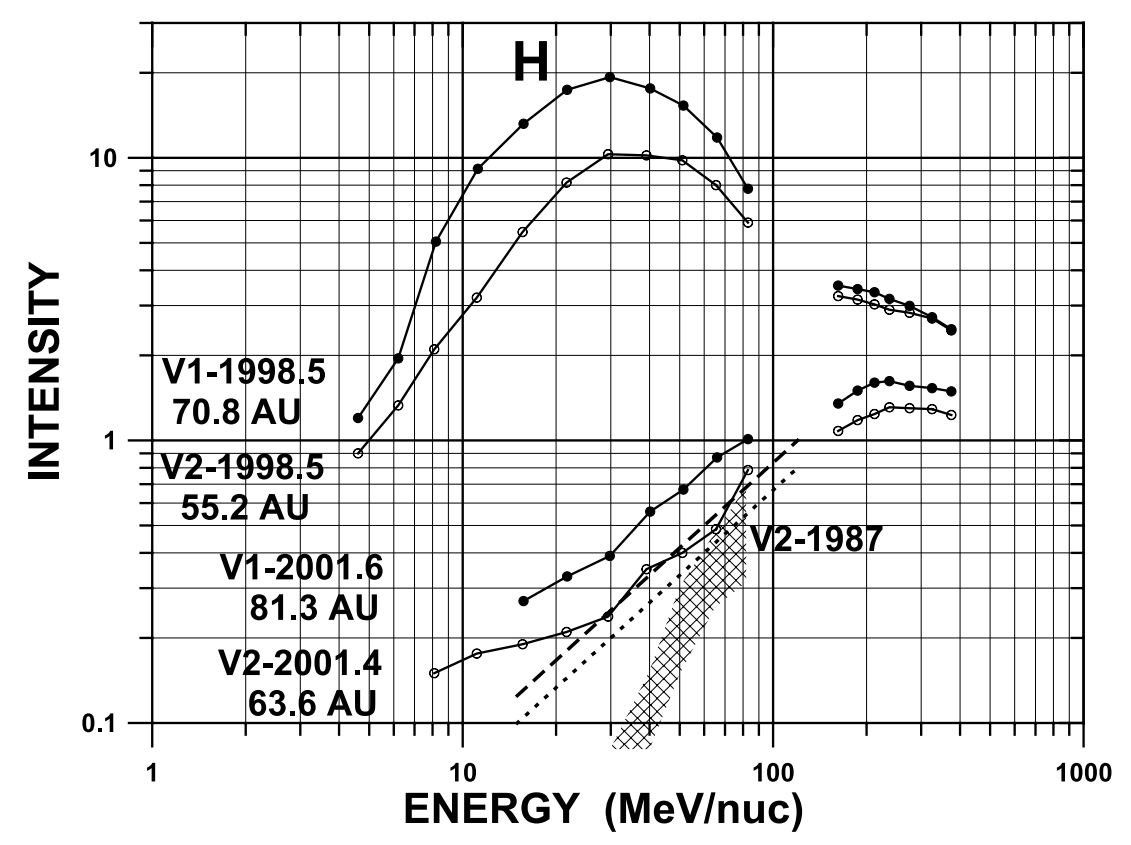

Figure 8. Measured total hydrogen spectra at V1 and V2 in 1998 during interval "2." Pair of dashed lines are estimated low-energy galactic spectra $\sim \mathrm{E}^{1.0}$ at V1 and V2 in interval " 2 ." Upper and lower limit anomalous H spectra in 1987 at 23 AU derived by Christian et al. [1988] shown as a shaded region. 


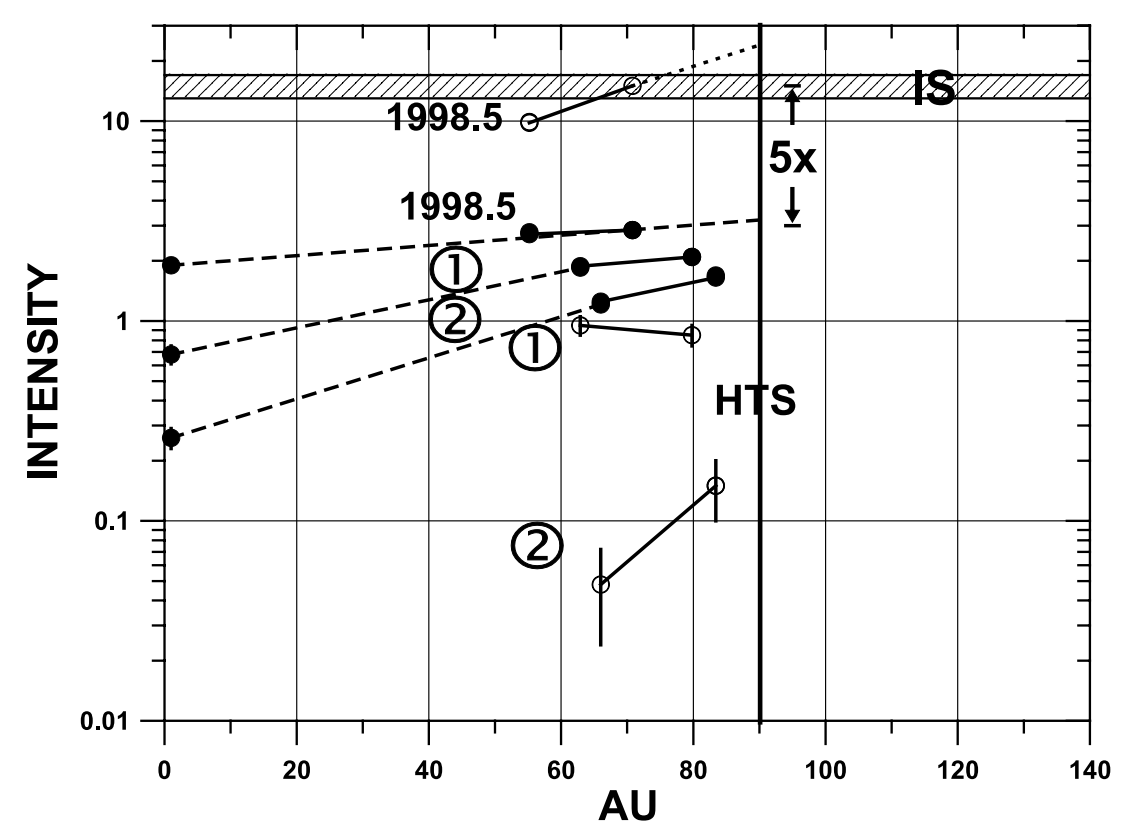

Figure 9. Radial profiles of intensities of $225 \mathrm{MeV}$ galactic $\mathrm{H}$ nuclei (solid circles) and 27-42 MeV anomalous H nuclei (open circles) observed at V1 and V2 as the modulation increases between 1998 and time intervals " 1 " and "2." Measurements at 1 AU for galactic H are also shown, connected to outer heliosphere measurements by dashed lines. Estimated interstellar intensity of $225 \mathrm{MeV}$ galactic $\mathrm{H}$ nuclei [Webber and Lockwood, 2001] is shown as a shaded region.

the intensity of $\mathrm{H}^{*}$ nuclei at V1 and V2 between 2000 and 2002. For $H^{*}$, if one examines carefully Figure 3 showing the intensity of 27-42 MeV H , and Figure 5 showing this intensity-corrected for galactic $\mathrm{H}$ nuclei and also Figure 8 showing the spectra at 1998.5 and again at interval " 2 ," that the $\mathrm{H}^{*}$ intensity in the $27-42 \mathrm{MeV}$ channel decreased by a factor of roughly 100 between 1998.5 and time period " 2 " at V2 and also by a factor $\sim 100$ at V1. During the same time the overall intensity decrease of higher-energy galactic cosmic rays was a factor $\sim 2.5$ at $\mathrm{V} 2$ and $\sim 2.0$ at V1. By 2002.0 the $\mathrm{H}^{*}$ intensity at all energies was even lower and essentially invisible at both V1 and V2 below the estimated galactic $\mathrm{H}$ spectrum as seen in Figure 4.

[11] In a recent paper, Webber and Lockwood [2004a] [see also McDonald et al., 2005; Van Allen and Randall, 2005] have discussed the overall galactic cosmic ray time variations during this time period at V1, V2, P10 and at the Earth. These temporal variations, which represent the 11year modulation in solar cycle 23, are seen to be largest at the Earth, smaller at V2 and still smaller at V1 where the overall magnitude of the intensity changes amounts to $\sim 50 \%$ or less even for the lowest-rigidity particles, $120-$ $230 \mathrm{MeV} \mathrm{H}$. These galactic particle intensity changes are confirmed in the present analysis.

[12] The anomalous particle intensity changes at V1 and V2 are potentially much more complex than those for galactic particles and may include intensity changes at their source, believed to be near the HTS, as well as the changing entry of these particles into the heliosphere owing to their changing drift patterns along the HTS. In addition, the $\mathrm{H}^{*}$ has a lower velocity and rigidity than the galactic $\mathrm{H}$ as well as a dramatically different spectrum which will affect the modulation through the Compton-Getting factor. We should note that V1 was already quite near the HTS in late 2002 at a distance of $\sim 86 \mathrm{AU}$ [Krimigis et al., 2003; McDonald et al., 2003a, 2003b], when the intensity changes described in this paper occurred.

[13] The total $\mathrm{H}$ spectra for the interval 1998 and time interval " 1 " is shown in Figure 7 and for 1998 and time interval " 2 " in Figure 8 as noted earlier. For illustration the anomalous $\mathrm{H}$ upper and lower limit spectra derived in 1987 from V2 data at $23 \mathrm{AU}$ in a previous negative polarity cycle are also shown in Figure 8 [Christian et al., 1988].

[14] To assist in the understanding of the intensity decrease of both galactic and anomalous $\mathrm{H}$ nuclei we also show in Figure 9 radial intensity profiles of $225 \mathrm{MeV}$ galactic $\mathrm{H}$ nuclei measured between $\mathrm{V} 1$ and $\mathrm{V} 2$ and the Earth for the three time intervals. For galactic $\mathrm{H}$ nuclei these radial profiles appear to steepen slightly with increasing modulation. This is consistent with the observation that the modulation of galactic $\mathrm{H}$ is larger at V2 than at V1 [e.g., Webber and Lockwood, 2004a]. It also emphasizes the fact that the measurements cover a range of radial distances that become a part of the overall modulation that is observed. Note that none of these galactic $\mathrm{H}$ nuclei radial intensity profiles in Figure 9 reasonably extrapolate to the estimated interstellar intensity $\sim 15 \pm 3 \mathrm{p} / \mathrm{m}^{2}$ sr s $\mathrm{seV}$ at this energy [Webber and Lockwood, 2001]. The extrapolated intensity at 90 AU for the 1998 radial profile is $\sim 3 \mathrm{p} / \mathrm{m}^{2}$ sr s MeV or about $1 / 5$ of the estimated IS intensity. This difference between the estimated galactic spectrum and that extrapolated to $90 \mathrm{AU}$, here referred to as the "outer heliosphere jump", in an earlier paper by Webber and Lockwood [2004b], is a measure of the overall magnitude of galactic particle modulation at this time and at this energy that is occurring at or beyond the HTS.

[15] In Figure 9 we also show the radial intensity profiles derived for 27-42 MeV anomalous $\mathrm{H}$ between V1 and V2. 
These intensities decrease much more dramatically between the observing periods than those for galactic $\mathrm{H}$, as we have noted earlier, but the radial intensity profiles themselves between V2 and V1 do not appear to become steeper as a result of this reduced intensity but instead remain roughly the same. In fact, these radial intensity profiles for anomalous $\mathrm{H}$ in intervals " 1 " and " 2 " extrapolated to $~ 90$ AU lie well below the 1998.5 profile extrapolated to $90 \mathrm{AU}$ suggesting that most of the large $\mathrm{H}^{*}$ modulation between 1998 and intervals " 1 " and " 2 " seems to be occurring not only beyond V1 but also beyond 90 AU.

\section{Models for Cosmic Ray Modulation and a Comparison With the Measurements in This Paper}

[16] As a result of new data in the last few years from both the Voyager and Pioneer spacecraft, which indicates a large modulation in the outermost heliosphere [Webber and Lockwood, 2001; McDonald et al., 2003a, 2003b; Van Allen and Randall, 2005], the most recent heliospheric cosmic ray modulation models now include the effects of an HTS embedded within a larger modulation region extending to an outer boundary at the heliopause, following the pioneering work of Jokipii et al. [1993] [e.g., Langner et al., 2003; Florinski et al., 2003; Potgieter and Langner, 2004; Caballero-Lopez et al., 2004]. For example, the model of Potgieter and Langner [2004] specifically calculates the expected intensities of both $\mathrm{H}^{*}$ and $\mathrm{H}$ nuclei as a function of radius and from these calculations one may obtain the modulation for $\mathrm{H}^{*}$ and $\mathrm{H}$ as the solar magnetic polarity changes and the heliospheric current sheet tilt changes from $10^{\circ}$ to $75^{\circ}$ thus providing the maximum 11 -year modulation. In this model the $\mathrm{H}$ intensity at $\sim 200 \mathrm{MeV}$ at the HTS is roughly a factor $\sim 5$ below the IS intensity at a time of minimum modulation in the positive polarity cycle (e.g., 1998). However, the curves presented in this paper for $\mathrm{H}^{*}$ modulation are not specific enough for our measurements between 2000 and 2002. The work of Caballero-Lopez et al. [2004] calculates only galactic H nuclei spectra. These calculations also give a reasonable fit to the data of galactic particles presented here. In particular they show that for the minimum modulation conditions in 1998, a decrease $\sim$ a factor of 5 is expected between the IS intensity and that at the HTS taken to be at 90 AU (e.g., modulation beyond the HTS). This is just the factor that we find the extrapolated intensity at $90 \mathrm{AU}$ to be less than the interstellar intensity, the so-called "outer heliosphere jump" in the work of Webber and Lockwood [2004b]. Florinski et al. [2003] have also presented detailed calculations of cosmic ray transport in a global heliosphere but their calculations do not directly compare with the energy $\sim 200 \mathrm{MeV}$ measured in this paper.

[17] A detailed comparison of these new Voyager measurements of anomalous $\mathrm{H}$ and model predictions is beyond the scope of this article. We believe that such comparisons need to be done by the modelers/theorists themselves.

\section{Summary and Conclusions}

[18] The disappearance of anomalous $\mathrm{H}$ nuclei and the corresponding 11-year modulation of galactic cosmic ray $\mathrm{H}$ nuclei at V1 and V2 in the outer heliosphere between 2000 and 2002 described in this paper provides an unprecedented insight into particle transport, drift and acceleration processes in the region near the HTS. In this case the absolute intensities and the amount of intensity change (modulation) can be followed for both of the galactic $\mathrm{H}$ and $\mathrm{H}^{*}$ species which are expected to have quite different source functions and spatial distributions. Because each species has $\mathrm{q} / \mathrm{A}=1$, however, aspects of the propagation within the heliosphere will be expected to be similarly organized with respect to velocity and rigidity.

[19] The results of this measurement using both V1 and V2 data can be summarized as follows:

[20] 1. For the galactic $H$ nuclei the overall intensity decreases observed between 1998 and 2002 are larger at V2 than at V1. This results in a steepening of the radial intensity profiles in the outer heliosphere as the modulation increases. These radial intensity profiles from 1 to $70-80$ AU at all three levels of modulation examined here extrapolate to roughly the same intensity at $\sim 90$ AU. This intensity is a factor $\sim 5$ below the estimated IS intensity at $225 \mathrm{MeV}$. This "outer heliosphere jump" represents a modulation that is occurring between the HTS and IS space outside of the modulating region.

[21] The rigidity dependence of the modulation within the heliosphere between 1998 and time intervals " 1 " and " 2 " for galactic $\mathrm{H}$ nuclei between 150 and $300 \mathrm{MeV}$ at both V2 and V1 is very similar to that observed for $\mathrm{H}$ nuclei near the Earth but the amplitude of this modulation is much smaller at the outer heliosphere spacecraft [e.g., Webber and Lockwood, 2004a].

[22] 2. For the anomalous $\mathrm{H}$ nuclei the fractional modulation between 1998 and intervals " 1 " and " 2 " is much larger than it is for galactic $\mathrm{H}$. The ratio between anomalous and galactic intensity changes is roughly a factor $\sim 20$ for both V1 and V2 data for the first two steps of the overall intensity decrease in 2000-2001. The overall decrease of anomalous $\mathrm{H}$ at both $\mathrm{V} 1$ and $\mathrm{V} 2$ is a factor $\sim 100$ during this time period. As a result of this very large modulation, the radial intensity profiles between V1 and V2 for $27-42 \mathrm{MeV}$ $\mathrm{H}^{*}$ get somewhat steeper as the modulation increases but do not extrapolate to a constant intensity at a fixed distance of 90 AU where the HTS might be located, for example. At the time of largest modulation at the end of 2001 the extrapolated intensity of $27-42 \mathrm{MeV} \mathrm{H}^{*}$ at $90 \mathrm{AU}$ is $<0.5 \%$ of what it was in 1998. This intensity decrease at a fixed distance occurring over a time period $\sim 1$ year could be the result of an intensity change of the source itself, but most likely is due to changes in the "connection" between V1 and V2 and the source itself, for example, due to the effects of changing drift and particle entry into the heliosphere during this time period.

[23] A final decrease of anomalous $\mathrm{H}$ in late 2001, when this component completely disappeared at both V1 and V2, has no obvious correlation with the intensity of galactic $\mathrm{H}$ at this time, which shows little change, and could be due to an entirely different cause.

[24] Acknowledgment. Zuyin Pu thanks Marty Lee, Gary P. Zank, and another reviewer for their assistance in evaluating this paper.

\section{References}

Caballero-Lopez, R. A., H. Moraal, and F. B. McDonald (2004), Galactic cosmic ray modulation: Effects of the solar wind termination shock and 
the heliosheath, J. Geophys. Res., 109, A05105, doi:10.1029/ 2003JA010358.

Christian, E., A. C. Cummings, and E. C. Stone (1988), Evidence for anomalous cosmic-ray hydrogen, Astrophys. J., 334, L77-L80.

Christian, E., A. C. Cummings, and E. C. Stone (1995), Observations of anomalous cosmic ray hydrogen from the Voyager spacecraft, Astrophys. $J ., 446$, L105-L108.

Fisk, L. A., B. Kozlovsky, and R. Ramaty (1974), An interpretation of the observed oxygen and nitrogen enhancements in low-energy cosmic rays, Astrophys. J., 190, L35-L37.

Florinski, V., G. P. Zank, and N. V. Pogorelov (2003), Galactic cosmic ray transport in the global heliosphere, J. Geophys. Res., 108(A6), 1228, doi:10.1029/2002JA009695.

Hill, M. E., D. C. Hamilton, J. E. Mazur, and S. M. Krimigis (2003), Anomalous cosmic ray intensity variations in the inner and outer heliosphere during the solar cycle 22 recovery phase $(1991-1999)$, J. Geophys. Res., 108(A10), 8037, doi:10.1029/2003JA009914.

Jokipii, J. R., J. Kota, and E. Merenyi (1993), The gradient of galactic cosmic rays at the solar wind termination shock, Astrophys. J., 405, $782-786$.

Krimigis, S. M., et al. (2003), Voyager 1 exited the solar wind at a distance $\sim 85$ AU from the Sun, Nature, 426, 45-48.

Langner, U. W., M. S. Potgieter, and W. R. Webber (2003), Modulation of cosmic ray protons in the heliosheath, J. Geophys. Res., 108(A20), 8039, doi:10.1029/2003JA009934.

McDonald, F. B., A. Lukasiak, and W. R. Webber (1995), Pioneer 10 and Voyager 1 observations of anomalous hydrogen in the outer heliosphere, Astrophys. J., 446, L101-L104.

McDonald, F. B., et al. (2000), The onset of the long-term cosmic ray modulation in cycle 23 coupled with a transient increase of anomalous cosmic rays in the distant heliosphere, J. Geophys. Res., 105, 20,99721,004 .

McDonald, F. B., A. C. Cummings, N. Lal, R. E. McGuire, and E. C. Stone (2001), Cosmic rays in the heliosphere over the solar minimum of cycle 22, Conf. Pap. Int. Cosmic Ray Conf. 27th.

McDonald, F. B., Z. Fujii, B. Heikkila, N. Lal, and R. F. McGuire (2003a), The radial distribution of galactic cosmic rays in the heliosphere at solar minimum and solar maximum, Conf. Pap. Int. Cosmic Ray Conf. 28th, $3965-3968$

McDonald, F. B., et al. (2003b), Enhancements of energetic particles near the heliospheric termination shock, Nature, 426, 48-51.

McDonald, F. B., et al. (2005), Cosmic ray observations in the heliosphere: 1972-2005, Conf. Pap. Int. Cosmic Ray Conf. 29th.

Potgieter, M. S., and U. W. Langner (2004), Modulation and acceleration of anomalous protons in the outer heliosphere, Adv. Space Res., 34, $132-$ 137.
Steenberg, C. D., A. C. Cummings, and E. C. Stone (1999), Drift calculations on the modulation of anomalous cosmic rays during the 1998 solar minimum period, Conf. Pap. Int. Cosmic Ray Conf., 26th.

Stone, E. C., A. C. Cummings, and W. R. Webber (1997), Evolution of the energy spectra of anomalous cosmic rays in the outer heliosphere, Conf. Pap. Int. Cosmic Ray Conf. 25th.

Stone, E. C., A. C. Cummings, D. C. Hamilton, M. E. Hill, and S. M. Krimigis (1999), Voyager observations of anomalous and galactic cosmic rays during 1998, Conf. Pap. Int. Cosmic Ray Conf. 26th.

Van Allen, J. A., and B. A. Randall (2005), Projected disappearance of the 11 -year cycle minimum of galactic cosmic ray intensity in the antapex direction within the outer heliosphere, Geophys. Res. Lett., 32, L07102, doi:10.1029/2005GL022629.

Webber, W. R., and J. A. Lockwood (2001), Voyager and Pioneer spacecraft measurements of cosmic ray intensities in the outer heliosphere: Toward a new paradigm for understanding the global solar modulation process: 1 Minimum solar modulation (1987 and 1997), J. Geophys. Res., 106, 29,323-29,331

Webber, W. R., and J. A. Lockwood (2004a), Onset and amplitude of the 11 -year solar modulation of cosmic ray intensities at the Earth and at Voyagers 1 and 2 during the period from 1997 to 2004, J. Geophys. Res., 109, A09103, doi:10.1029/2004JA010492.

Webber, W. R., and J. A. Lockwood (2004b), Heliocentric radial intensity profiles of galactic cosmic rays measured by the IMP, Voyager, and Pioneer spacecraft in solar 11-year modulation cycles of opposite magnetic polarity, J. Geophys. Res., 109, A11101, doi:10.1029/ 2004JA010642.

Webber, W. R., F. B. McDonald, J. A. Lockwood, and B. Heikkila (2002), The effect of the July 14, 2000, Bastille Day solar flare event on $>70 \mathrm{MeV}$ galactic cosmic rays observed at V1 and V2 in the distant heliosphere, Geophys. Res. Lett., 29(10), 1377, doi:10.1029/2002GL014729.

Webber, W. R., A. C. Cummings, E. C. Stone, F. B. McDonald, N. Lal, and B. Heikkila (2005), Differences in the spectra of anomalous cosmic ray helium nuclei in two solar magnetic polarity cycles, J. Geophys. Res., 110, A07106, doi:10.1029/2005JA011123.

A. C. Cummings and E. C. Stone, Downs Laboratory, California Institute of Technology, Pasadena, CA 91125, USA.

B. Heikkila and N. Lal, NASA Goddard Space Flight Center, Greenbelt, MD 20771, USA.

F. B. McDonald, Institute of Physical Science and Technology, University of Maryland, College Park, MD 20742, USA.

W. R. Webber, Department of Astronomy, New Mexico State University, Las Cruces, NM 88003, USA. (bwebber@nmsu.edu) 